\title{
Violência contra crianças e adolescentes - proposta de classificação dos níveis de gravidade
}

\author{
Child and adolescent physical abuse - a proposal for classifying its severity
}

Luci Pfeiffer ${ }^{1}$, Nelson Augusto Rosário ${ }^{2}$, Monica Nunes L. Cat ${ }^{3}$

\section{RESUMO}

Objetivo: Descrever as formas de apresentação da violência e método de classificação em níveis de gravidade aplicado no Programa Rede de Proteção das Crianças e Adolescentes em Situação de Risco para Violência da cidade de Curitiba.

Métodos: O presente estudo constituiu-se de quatro fases: participação e acompanhamento da implantação do programa Rede de Proteção; desenvolvimento de método para classificar os níveis de gravidade das situações de violência contra infância e adolescência; participação na capacitação de profissionais da rede pública para identificação das situações de violência, aplicação do método de classificação e notificação; análise e interpretação dos dados das notificações de violência geradas pelo Programa.

Resultados: No Programa Rede de Proteção de Curitiba foram notificados 1.537 casos de violência contra crianças e adolescentes em 2003 e 1.974 em 2004, sendo 93\% casos de violência doméstica. Dos casos de violência doméstica, em 2003 e 2004, respectivamente, 40,7 e 61,7\% foram situações de negligência, 35,2 e 26\%, de violência física; 17,5 e 8,8\%, sexual e 6,6 e 3,5\%, psicológica isolada. Não houve diferença significativa entre sexo da vítima, com predomínio na faixa etária de cinco a nove anos. Em 2003, os casos moderados corresponderam a $41,8 \%$, os graves, a $40 \%$ e os leves, a 18,2\%. Em 2004, 35,4\% foram casos moderados, $33,9 \%$, leves e $30,7 \%$, graves.

Conclusões: Este estudo apresenta aspectos e interfaces da violência contra crianças e adolescentes e a aplicação de novo método de classificação de seus níveis de gravidade, o que tornou possível estabelecer critérios de urgência e emergência, além da estruturação de fluxogramas e protocolos de atendimento de acordo com esses níveis.

Palavras-chave: violência; criança; adolescente; violência doméstica; maus-tratos infantis; epidemiologia.

\section{ABSTRACT}

Objective: To report different clinical presentation of child physical abuse and propose a method for classifying its severity as applied to a program that provides assistance for children and adolescents under risk in Curitiba, in Southern Brazil.

Methods: The study comprised four phases: involvement and assessment of the implementation of the network that provides assistance for children and adolescents under risk; development of a method to classify abuse according to its severity level in childhood and adolescence; training health professionals to identify beforehand cases of abuse and how to apply the proposed classification method and to notify the authorities; analysis and interpretation of collected data.

Results: 1,537 cases were notified in 2003 and 1,972 in 2004; $93 \%$ of them were considered as domestic abuse. In those cases of domestic abuse, in 2003 and 2004 respectively, negligence was reported in 40.7 and $61.7 \%$; physical violence occurred in 35.1 and $26 \%$, sexual abuse in 17.6 and $7.7 \%$ sexual abuse, and psychological stress in 6.6 and $4.6 \%$. Gender was not significantly different among victimized
Instituição: Departamento de Pediatria da Universidade Federal do Paraná (UFPR), Curitiba, PR, Brasil

'Mestre em Saúde da Criança e do Adolescente pela UFPR; Responsável pelo Programa de Defesa dos Direitos da Criança e do Adolescente do HC-DEDICA da UFPR, Curitiba, PR, Brasil

2Doutor em Saúde da Criança e do Adolescente pela Universidade Estadual de Campinas (Unicamp); Professor Titular da UFPR, Curitiba, PR, Brasil ${ }^{3}$ Doutora em Pediatria pela UFPR; Professora Adjunta da UFPR, Curitiba, PR, Brasil

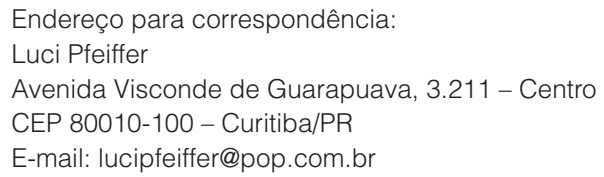

Endereço para correspondência:

Luci Pfeiffer

Avenida Visconde de Guarapuava, 3.211 - Centro

CEP 80010-100 - Curitiba/PR

E-mail: lucipfeiffer@pop.com.br

Conflito de interesse: nada a declarar

Recebido em: 28/3/2010

Aprovado em: 21/2/2011 
subjects, but the main age interval among victims was five to nine years old. In 2003, severity was classified as mild in $8.2 \%$, moderate in $41.8 \%$, and severe $40 \%$. In the following year, $33.9 \%$ of the cases were identified as mild, $35.4 \%$ as moderate, and $30.7 \%$ as severe.

Conclusions: This study shows the features and interfaces of abuse against children and adolescents, as well as the implementation of a new method for severity classification, which allowed to establish criteria for emergency care and to create flowcharts and individualized treatment protocols according with these levels.

Key-words: violence; child; adolescent; domestic violence; child abuse; epidemiology.

\section{Introdução}

É possível definir a violência contra crianças e adolescentes como toda ação ou omissão - provocada por pessoa de maturidade física e psicológica mais adiantada - que venha a lhes provocar qualquer forma de dor. Até os 18 anos, as situações mais comuns de violência são classificadas como doméstica, intrafamiliar ou maus-tratos, que se caracterizam quando essa ação ou omissão é praticada por adulto ou adolescente com maturidade física e psíquica mais adiantada, que exerça sobre a vítima o papel de cuidador ou responsável, seja por paternidade, outro vínculo de parentesco, dependência, convivência, afeto ou confiança. Habitualmente, a violência ocorre dentro das próprias casas - sendo os agressores mais frequentes os pais ou aqueles que ocupam seu lugar, seguidos por outros do núcleo de convivência da criança ${ }^{(1)}$ - podendo se apresentar nas formas mais diversas e bizarras, em variados níveis de gravidade ${ }^{(2)}$.

A partir de 1990, com o Estatuto da Criança e do Adolescente no Brasil, passou-se a ter instrumentos legais de proteção nas situações de violência na infância e na adolescência, tornando obrigatória sua notificação (artigo 245), até mesmo de mera suspeita ${ }^{(3)}$. O Código Penal Brasileiro, em artigos escritos em 1940, como os de número 129 e 136, define e estipula os critérios de diagnóstico e classificação em níveis de gravidade para as lesões corporais e maustratos, respectivamente, ignorando os danos psicológicos e minimizando as situações de negligência ${ }^{(4,5)}$. Em 2009, foi promulgada a lei de número 12.015, que modifica a anterior ao tratar dos crimes sexuais, antes ditos como "Crimes contra os Costumes" e hoje, "Crimes contra a Dignidade Sexual”. Nesta, é introduzida, no artigo 217 A, a figura do "Estupro de Vulnerável", definindo-se como crime qualquer ato sexual praticado contra pessoa com idade até $14 \operatorname{anos}^{(6,7)}$.

No entanto, em nosso país, os registros das situações de violência na infância e na adolescência ainda são poucos e não há como se falar em epidemiologia, ainda que o Ministério da Saúde, em 2001, por meio da portaria n. 737, tenha definindo ser este não apenas um problema social, mas também - e, principalmente -, de Saúde Pública e de notificação obrigatória em todo território nacional ${ }^{(8)}$ (Política Nacional de Redução de Morbimortalidade por Acidentes e Violência). Porém, as denúncias e notificações têm se mantido em números infinitamente inferiores à realidade esperada ${ }^{(9)}$.

Em Curitiba, iniciou-se, no ano de 1998, um trabalho de enfrentamento da violência, com a união de esforços das diversas Secretarias, Programas, organizações não governamentais voltadas à área da infância e da adolescência, bem como da Sociedade Paranaense de Pediatria. Formulou-se uma ficha de notificação única e padronizada e instituiu-se um trabalho de capacitação para os profissionais que atuam com crianças e adolescentes para prevenção, diagnóstico, avaliação do nível de gravidade de cada caso, notificação e acompanhamento das situações de risco para violência. Desde 2002, as notificações passaram a ser analisadas e armazenadas em um banco de dados da Secretaria Municipal de Saúde, instituindo-se o Programa Rede de Proteção das Crianças e Adolescentes em Situação de Risco para Violência.

O presente trabalho objetivou descrever a violência contra crianças e adolescentes na cidade de Curitiba, por meio das notificações ao Programa Rede de Proteção das Crianças e Adolescentes em Situação de Risco para Violência, além de relatar o desenvolvimento de um sistema de classificação em níveis de gravidade para definir o fluxograma de atendimento e a necessidade de medidas emergenciais de proteção.

\section{Método}

A partir da implantação de amplo programa de enfrentamento à violência contra crianças e adolescentes de Curitiba, foi possível obter dados referentes às suas formas de apresentação, bem como sua natureza e frequência segundo idade e sexo. Da análise do primeiro ano do programa de notificação, em 2001, surgiu tanto a necessidade de estabelecer um fluxo de atendimento quanto definir situações de maior ou menor gravidade. 
Assim, iniciou-se o presente trabalho, com a implantação do programa Rede de Proteção às Crianças e Adolescentes em Situação de Risco para a Violência da cidade de Curitiba, que se deu em várias fases: idealização, elaboração e criação da ficha de notificação (1998-2000), capacitação de profissionais da rede pública (cerca de dez mil), das áreas da saúde, educação, ação social, justiça e segurança, para identificação e notificação obrigatória (2000-2002), acompanhamento dos casos e desencadeamento das medidas de proteção (2003 até o momento atual).

O presente estudo constituiu-se, então, de quatro fases: 1) participação e acompanhamento da implantação do programa; 2) desenvolvimento de um método para classificar os níveis de gravidade das situações de violência contra a infância e a adolescência, durante a etapa da estruturação do programa; 3) participação na capacitação de profissionais da rede pública para indentificar situações de violência, com aplicação do método de classificação e notificação; 4) análise dos dados das fichas de notificação de violência contra crianças e adolescentes, armazenadas no banco de dados da Secretaria Municipal de Saúde, provenientes das instituições públicas municipais de atendimento às crianças e aos adolescentes, de hospitais e de Conselhos Tutelares, referentes aos anos de 2003 e 2004.

Foram incluídos todos os casos notificados de violência contra crianças e adolescentes no período de 2003 (1.537) e 2004 (1.974), sendo o tamanho da amostra o total dessas notificações. Foram excluídas, no tocante à apreciação dos níveis de gravidade, 256 notificações em 2003 e 244 em 2004 que não continham essa informação.

O método desenvolvido para classificar as situações de violência na infância e na adolescência em níveis de gravidade foi composto a partir da eleição, por maior frequência nas notificações de 2001, de quatro determinantes de morbidade nessas situações, a saber: o estado geral da vítima, a constituição familiar e seu posicionamento frente à violência apresentada, o tipo e as características da agressão e o perfil do agressor (Tabela 1). Por meio da soma do risco encontrado para cada fator, conforme indicadores específicos, os casos foram classificados em:

- Sem evidências de violência ( 0 a 3 pontos): os sinais e sintomas trazidos pela criança não se enquadram nas características das lesões físicas intencionais, violência psicológica, sexual ou negligência; sem identificação de agressão e agressor, com a presença de laços de respeito, confiança e afeto bem estabelecidos entre a criança e responsáveis.
- Caso leve ( 4 a 5 pontos): presença de sinais ou sintomas compatíveis com um episódio único de agressão física, psicológica ou detecção de negligência, sem consequências à saúde física ou mental da vítima, com responsáveis dispostos a rever sua conduta agressiva.

- Caso moderado (6 a 8 pontos): sinais ou sintomas que demonstram violência física, psicológica e/ou negligência que possam levar a danos físicos ou emocionais e que necessitem de tratamento ambulatorial e acompanhamento especializado tanto da vítima como dos agressores, ou que se apresentem como leves, mas de repetição.

- Caso grave (9 a 11 pontos): situações de abuso sexual ou violência física, psicológica e/ou negligência; a vítima apresenta marcas físicas ou emocionais da violência sofrida, com necessidade de atendimento hospitalar e/ou especializado, incluindo saúde mental, sem demonstração clara por parte dos responsáveis quanto ao interesse pelo tratamento e/ou à mudança de suas atitudes agressivas.

- Caso com risco de morte (12 pontos): situações de abuso sexual por parte de responsáveis ou pessoas de convivência da vítima, violência física, psicológica e/ou negligência que necessitam de tratamento terciário, especializado e emergencial, incluindo saúde mental, com sinais de participação, conivência e/ou impotência da família tanto nuclear como expandida.

Para o tratamento estatístico, foram aplicados os testes não paramétricos: qui-quadrado de Pearson, qui-quadrado de Pearson com correção de Yates e qui-quadrado para tendência linear, considerando-se nível de significância de 5\% e poder de teste mínimo de $90 \%$. Esta pesquisa foi aprovada pelo Comitê de Ética e Pesquisa em Seres Humanos do Hospital de Clínicas da Universidade Federal do Paraná e da Secretaria Municipal de Saúde de Curitiba.

Tabela 1 - Método de classificação dos níveis de gravidade da violência contra crianças e adolescentes

\begin{tabular}{lccccc}
\hline & Vítima & Agressão & Agressor & Família & Limiares \\
\hline Ausente & 0 & 0 & 0 & $0-3$ & 0 a 3 \\
Leve & 1 & 1 & $1-2$ & $1-2$ & 4 a 5 \\
Moderado & $1-2$ & $1-2$ & 2 & $1-2-3$ & 6 a 8 \\
$\begin{array}{l}\text { Grave } \\
\begin{array}{l}\text { Risco de } \\
\text { morte }\end{array}\end{array}$ & $3-3$ & $2-3$ & $2-3$ & $1-2-3$ & 9 a 11 \\
\end{tabular}




\section{Resultados}

Em 2003 e 2004, foram registradas 1.537 e 1.974 notificações, respectivamente, pelas 643 unidades de assistência a crianças e adolescentes nas áreas da saúde, educação e ação social que participam do programa Rede de Proteção em Curitiba. A natureza preponderante da violência em 2003 foi a doméstica ou maus-tratos, com $92,6 \%$ das notificações, sendo as demais $(7,4 \%)$ relacionadas à violência extrafamiliar. No ano de 2004, observou-se proporção semelhante, com $92,9 \%$ dos casos de violência doméstica e $7,1 \%$ de extrafamiliar.

Quanto às vítimas, a maior incidência ocorreu na faixa etária de 5-9 anos, nos dois anos estudados. Seguiu-se a essa faixa a de crianças de $0-4$ anos, das quais 10,9\% referem-se a lactentes menores de um ano de idade, incluindo 64 crianças com menos de um mês de vida. Cerca de um quarto dos casos corresponderam às idades de dez a 14 anos (Tabela 2).

No que tange à agressão, negligência ou omissão do cuidar foi responsável pelo maior número de notificações, com incidência significativamente maior do que os outros tipos de violência $(p<0,01)$. A violência física teve frequência

Tabela 2 - Número e porcentual de notificações de casos de violência segundo a faixa etária da vítima

\begin{tabular}{lrrrr}
\hline Faixa etária & \multicolumn{2}{c}{$\mathbf{2 0 0 3}$} & \multicolumn{2}{c}{$\mathbf{2 0 0 4}$} \\
\cline { 2 - 5 } (anos) & $\mathbf{n}$ & \multicolumn{1}{c}{$\%$} & \multicolumn{1}{c}{$\mathbf{n}$} & \multicolumn{1}{c}{$\%$} \\
\hline Antes do & 6 & 0,4 & 10 & 0,5 \\
nascimento & & & & \\
0 a 4 & 404 & 29,8 & 572 & 31,2 \\
5 a 9 & 520 & 38,5 & 664 & 36,2 \\
10 a 14 & 346 & 25,6 & 480 & 26,2 \\
15 a 18 & 77 & 5,7 & 108 & 5,9 \\
Total & $\mathbf{1 3 5 3}$ & $\mathbf{1 0 0 , 0}$ & $\mathbf{1 8 3 4}$ & $\mathbf{1 0 0 , 0}$ \\
\hline
\end{tabular}

Fonte: Adaptado de CE/SMS de Curitiba - Banco de dados da Rede de Proteção - 2003/2004. significativamente maior do que a sexual e psicológica $(p<0,01)$, como pode ser observado na Tabela 2 .

Não houve diferença significativa da incidência global da violência de acordo com o sexo da vítima. Entretanto, a negligência e a violência física foram mais frequentes no sexo masculino nos dois períodos estudados. Já no sexo feminino, a violência sexual correspondeu a cerca de três quartos dos casos (Tabela 3).

A mãe foi o agente agressor mais frequente, sendo responsável por 44,6\% dos casos em 2003 e 59,5\% em 2004 . O pai ocupou o segundo lugar, com $25,6 \%$ das notificações em 2003 e 23,4\% em 2004. A autoagressão foi o motivo de 13 notificações em 2003 e 11 em 2004. Especificamente nos casos de violência sexual, o padrasto ou companheiro da mãe ocupou o primeiro lugar, seguido pelo pai, avô, tio e outros com quem a vítima mantinha laços de dependência, afeto ou convivência. Em 5,3\% dos casos de abuso sexual, o agressor era pessoa conhecida da vítima, mas sem vínculos de moradia ou dependência direta e, em 3,3\% das notificações, o agressor era totalmente desconhecido da vítima.

$\mathrm{Na}$ avaliação das 1.281 notificações que continham informação sobre os níveis de gravidade no ano de 2003, os casos moderados foram os mais frequentes, seguidos pelos graves $\mathrm{e}$ leves (Tabela 3). Não se configurou diferença significante na frequência dos casos graves e moderados, mas o número de casos leves em 2003 foi expressivamente menor $(p<0,001)$. Das 1.730 notificações em 2004, os casos classificados como moderados foram os de maior incidência, seguidos dos leves e graves, não havendo diferença significativa entre os casos leves e moderados. A frequência de casos moderados foi significativamente superior aos graves $(p=0,001)$ (Tabela 4 ).

Ao analisar o nível de gravidade e tipos de violência, os casos de negligência e de violência física foram, em sua maioria, classificados como leves ou moderados $(p<0,001)$; já os de violência sexual foram predominantemente graves

Tabela 3 - Número e percentual de notificações segundo o tipo de violência e sexo

\begin{tabular}{|c|c|c|c|c|c|c|c|c|c|c|c|c|}
\hline & \multicolumn{6}{|c|}{2003} & \multicolumn{6}{|c|}{2004} \\
\hline & \multicolumn{2}{|c|}{ Feminino } & \multicolumn{2}{|c|}{ Masculino } & \multicolumn{2}{|c|}{ Total } & \multicolumn{2}{|c|}{ Feminino } & \multicolumn{2}{|c|}{ Masculino } & \multicolumn{2}{|c|}{ Total } \\
\hline & $\mathrm{n}$ & $\%$ & $\mathbf{n}$ & $\%$ & $\mathbf{n}$ & $\%$ & $\mathbf{n}$ & $\%$ & $\mathbf{n}$ & $\%$ & $\mathbf{n}$ & $\%$ \\
\hline Negligência & 248 & 44,9 & 304 & 55,1 & 552 & 40,7 & 532 & 47,0 & 599 & 53,0 & 1.131 & 61,7 \\
\hline Física & 225 & 47,3 & 251 & 52,7 & 476 & 35,2 & 237 & 49,7 & 240 & 50,3 & 477 & 26,0 \\
\hline Sexual & 180 & 75,6 & 58 & 24,4 & 238 & 17,5 & 118 & 73,3 & 43 & 26,7 & 161 & 8,8 \\
\hline Psicológica & 44 & 48,9 & 46 & 49,1 & 90 & 6,6 & 42 & 64,6 & 23 & 35,4 & 65 & 3,5 \\
\hline Total & 697 & 51,4 & 659 & 48,6 & 1356 & 100,0 & 929 & 50,6 & 905 & 49,4 & 1834 & 100,0 \\
\hline
\end{tabular}

Fonte: Adaptado do Banco de Dados do CE/SMS. Rede de Proteção, Curitiba, 2003/2004 
$(p<0,001)$. Para a violência psicológica, houve maior incidência do nível moderado $(p=0,03)$.

\section{Discussão}

De acordo com estudo desenvolvido com base nos dados das fichas de notificação das situações de violência contra crianças e adolescentes na cidade de Curitiba, nos anos 2003 e 2004, verificou-se que, dentre as situações de violência na infância e na adolescência, tiveram maior frequência as de natureza doméstica, caracterizando o crime de maus-tratos, sendo os principais agressores os próprios pais ou aqueles que ocupam seu lugar. Essa é uma constatação presente em muitos outros estudos internacionais, como de Kaplan e Sadock, que apontam os pais como responsáveis pela violência contra seus filhos em $75 \%$ dos casos, seguidos por outros parentes e cuidadores ${ }^{(10)}$.

Segundo relatórios da Unicef, a OMS estima que, em 2002, cerca de 53 mil crianças morreram em todo mundo em decorrência de homicídio ${ }^{(11)}$ e que $20 \%$ das mulheres e $10 \%$ dos homens de todo o mundo tenham sofrido alguma forma de violência na infância ${ }^{(12)}$.

No que se refere à faixa etária, observou-se maior frequência na de 5-9 anos, seguida pela faixa de 0-4 anos, impressionando a ocorrência de um quarto desses casos em lactentes com menos de um ano de idade, que tiveram suas mães como o agressor mais identificado.

De acordo com o relatório mundial sobre trabalho infantil (OIT 2002), em todo o mundo há 245 milhões de pessoas menores de 18 anos que trabalham. Desse total, mais de 186 milhões estão na faixa etária de cinco a 14 anos e 59 milhões entre 15 e 17 anos. Aproximadamente 170 milhões trabalham em condições perigosas. Cerca de dois milhões de crianças e adolescentes em todo o mundo se encontram em situação de exploração sexual comercial e pornografia ${ }^{(13)}$. No

Tabela 4 - Número e percentual de notificações segundo os níveis de gravidade

\begin{tabular}{lrrrr}
\hline & \multicolumn{2}{c}{$\mathbf{2 0 0 3}$} & \multicolumn{2}{c}{$\mathbf{2 0 0 4}$} \\
\cline { 2 - 5 } & $\mathbf{n}$ & $\mathbf{\%}$ & $\mathbf{n}$ & \multicolumn{1}{c}{3} \\
\hline Leve & 233 & 18,2 & 587 & 33,9 \\
Moderado & 535 & 41,8 & 613 & 35,4 \\
Grave & 513 & 40,0 & 530 & 30,7 \\
Total & $\mathbf{1 2 8 1}$ & $\mathbf{1 0 0 , 0}$ & $\mathbf{1 7 3 0}$ & $\mathbf{1 0 0 , 0}$ \\
\hline
\end{tabular}

Fonte: Adaptado do Banco de Dados, CE/SMS. Rede de Proteção, Curitiba, 2003/2004

$p=0,60$.
Brasil, estimativas indicam que cerca de 500 mil crianças, na faixa etária de nove a 17 anos, são exploradas sexualmente no país ${ }^{(14)}$.

Em países com sistemas de notificação obrigatória implantados há mais de três décadas, como o US Department of Health and Human Services, o abuso físico é prevalente em crianças abaixo de cinco anos (32\%), mas também há grande incidência entre 5-9 (27\%) e 10-14 (27\%) anos ${ }^{(15)}$. Dados da Organização para Cooperação e Desenvolvimento Econômicos (OCDE) indicam que, no universo de indivíduos com menos de 18 anos de idade, os bebês de até um ano são o grupo que corre o segundo maior risco de morte por homicídio. O risco de morte é cerca de três vezes maior para crianças com menos de um ano de idade do que para o grupo entre 1-4 anos de idade; e, quanto mais nova a criança, maior será a probabilidade de sua morte ser causada por um parente próximo ${ }^{(16)}$.

Ressalta-se que as situações de violência, especialmente as cometidas pelos pais ou responsáveis, têm efeito deletério duplo na infância e na adolescência, tanto pelo ato em si que vai ficar registrado na memória, independentemente da idade em que ocorre -, como pela quebra do estímulo ao desenvolvimento. Os traumas que excedem a capacidade de compreensão e elaboração da criança e do adolescente podem ter efeitos imediatos ou tardios, que repercutirão no seu modo primário de pensar e de agir, bem como na sua capacidade de enfrentar outras situações de dificuldades ou estresse para o resto de suas vidas ${ }^{(17)}$. Quanto menor a idade da vítima e maior o vínculo com o agressor, piores as possibilidades de sequelas, haja vista que crianças com menos de três anos de idade tendem a não produzir uma recordação verbal de traumas ou abusos passados, dificultando o diagnóstico. Contudo, suas experiências podem ser reproduzidas por meio de jogos, desenhos ou fantasias ${ }^{(18)}$. Ainda, adultos que foram abusados fisicamente na infância têm maior probabilidade de abusarem de seus filhos, comparados àqueles que não sofreram essa forma de violência ${ }^{(19)}$.

O tipo de violência mais notificada no Programa Rede de Proteção foi a negligência, seguido pela violência física, sexual e psicológica, sendo este último diagnóstico uma evolução na interpretação das várias apresentações da violência na infância e na adolescência.

Houve uma frequência geral semelhante para os sexos feminino e masculino, diferenciando-se, no entanto, quanto ao tipo de violência, com predomínio da negligência e da violência física para o sexo masculino e do abuso sexual para o feminino. 
Quanto ao nível de gravidade, em 2003, houve um maior número de casos classificados como moderados, seguidos pelos graves e, em menor porcentagem, ocorreram os leves. Em 2004, manteve-se a maior incidência de casos moderados, com acréscimo das notificações de casos leves, seguidas pelos graves. A aplicação de método classificatório da gravidade tornou possível estabelecer critérios de urgência e emergência, além de protocolos de acompanhamento de acordo com o risco geral da situação de violência. Da mesma forma, permitiu a criação de fluxos e a definição da complexidade de tratamento e das medidas de proteção legais e sociais no Programa Rede de Proteção. Novos estudos estão sendo desenvolvidos para validar o método, a fim de ampliar seu uso como instrumento de triagem na assistência a crianças e adolescentes em situação de risco para violência.

Vale ressaltar que os dados levantados das fichas de notificação obrigatória de situações de risco para violência

\section{Referências bibliográficas}

1. Pfeiffer $L$, Waksman RD. Injúrias intencionais. In: Campos Júnior $D$. Tratado de pediatria. São Paulo: Manole; 2006. p. 129.

2. Pfeiffer $L$, Waksman RD. Violência na infância e adolescência. In: Campos JA, Paes CEN, Blank D, Costa DM, Pfeiffer L, Waksman RD, editors. Manual de Segurança da Criança e do Adolescente. Belo Horizonte: Sociedade Brasileira de Pediatria; 2004. p. 95-278.

3. Brasil - Presidência da República. Estatuto da Criança e do Adolescente. Brasília: Presidência da República; 1990.

4. Prado LR. Artigo 129 do Código Penal Brasileiro. In: Prado LR. Comentários ao Código Penal. $4^{\mathrm{a}}$ ed. São Paulo: Revista dos Tribunais; 2007. p. 456-62.

5. Prado LR. Artigo 136 do Código Penal Brasileiro. In: Prado LR. Comentários ao Código Penal. $4^{\text {a }}$ ed. São Paulo: Revista dos Tribunais; 2007. p. 488-92.

6. Brasil. Presidência da República. Casa Civil [homepage on the Internet]. Lei 12.015/2009 (Lei Ordinária) de 7 de agosto de 2009 [cited 2010 May 23]. Available from: http://www.planalto.gov.br/ccivil_03/_Ato2007-2010/2009/Lei/ L12015.htm

7. JusBrasil. Legislação [homepage on the Internet]. Lei 12015/09 | Lei $n^{\circ}$ 12.015, de 7 de agosto de 2009 [cited 2010 May 23]. Available from: http:// www.jusbrasil.com.br/legislacao/818585/lei-12015-09

8. Brasil - Ministério da Saúde. Política nacional de redução de morbimortalidade por acidentes e violências. Portaria GM/MS No 737 de 16/05/01. Publicada no DOU n 96, Seção 1e - de 18/05/01. Brasília: MS/Opas; 2001.

9. Pfeiffer L, Pires JM. Principais tipos de violência. In: Sociedade Brasileira de Pediatria. Crianças e adolescentes seguros. São Paulo: Publifolha; 2006. p. 253.

10. Sadock BJ, Sadock VA. Problemas relacionados ao abuso e negligência. In: Sadock BJ, Sadock VA. Compêndio de psiquiatria. $9^{\text {a }}$ ed. Porto Alegre: Artmed; 2007. p. 940. na infância e na adolescência correspondem a uma parcela das vítimas, as que tiveram o diagnóstico de violência nos serviços públicos de atendimento à saúde, de educação e de proteção social. Há que se levar em conta que outras crianças e adolescentes que não frequentam essa rede pública podem permanecer sem diagnóstico e, portanto, este trabalho apresenta um perfil epidemiológico da violência nessa faixa etária, não podendo caracterizar índices gerais de prevalência ou incidência de tal entidade. No entanto, o estudo apresentado traz à tona as diversas apresentações da violência contra crianças e adolescentes, evidenciando sua ocorrência em todas as faixas etárias, sem distinção de sexo. O estudo apresenta ainda a possibilidade de utilizar um método para classificar os níveis de gravidade dessa violência, com vistas a sustentar a estruturação de fluxogramas e protocolos de atendimento, de acordo com a necessidade de cada caso.

11. UNICEF [homepage on the Internet]. Relatório: Estudo sobre a Violência contra Crianças: Brasil; 2005. p. 10-1 [cited 2010 May 23]. Available from: http://www.unicef.org/brazil/pt/Estudo_PSP_Portugues.pdf

12. UNICEF. Violência: relatório situação da infância brasileira. Brasil: UNICEF; 2006.

13. Organização Internacional para Erradicação do Trabalho Infantil [homepage on the Internet]. Combate à exploração sexual comercial de crianças: guia para educadores [cited 2010 May 23]. Available from: http://www.white.oit. org.pe/ipec/documentos/guia_educadores_br.pdf

14. IPE [homepage on the Internet]. Experiências de combate à exploração sexual comercial infantil no brasil. documento: boas práticas de combate ao trabalho infantil [cited 2010 May 23]. Available from: http://www.ilo.org/public/portugue/ region/ampro/brasilia/dezanos/pdf/sexual.pdf

15. U.S. Department of Health and Human Services, Administration on Children, Youth and Families. Child maltreatment - 2005. Washington, DC: U.S. Government Printing Office; 2007.

16. Bryce J, Terreri N, Victora CG, Mason E, Daelmans B, Bhutta ZA et al. Countdown to 2015: tracking intervention coverage for child survival. Lancet 2006;368:1067-76.

17. Cardon L, Pfeiffer L. Conseqüência dos traumas na infância e adolescência. In: Sociedade Brasileira de Pediatria. Crianças e adolescentes seguros. São Paulo: Publifolha; 2006. p. 292.

18. Kellogg ND; Committee on Child Abuse and Neglect. Evaluation of suspected child physical abuse. Pediatrics 2007;119:1232-41.

19. Ertem IO, Leventhal JM, Dobbs S. Intergenerational continuity of child physical abuse: how good is the evidence? Lancet 2000;356:814-9. 